TRANSACTIONS OF THE

AMERICAN MATHEMATICAL SOCIETY

Volume 352, Number 5, Pages 2369-2388

S 0002-9947(00)02579-4

Article electronically published on February 14, 2000

\title{
STATISTICAL PROPERTIES FOR NONHYPERBOLIC MAPS WITH FINITE RANGE STRUCTURE
}

\author{
MICHIKO YURI
}

\begin{abstract}
We establish the central limit theorem and non-central limit theorems for maps admitting indifferent periodic points (the so-called intermittent maps). We also give a large class of Darling-Kac sets for intermittent maps admitting infinite invariant measures. The essential issue for the central limit theorem is to clarify the speed of $L^{1}$-convergence of iterated Perron-Frobenius operators for multi-dimensional maps which satisfy Renyi's condition but fail to satisfy the uniformly expanding property. Multi-dimensional intermittent maps typically admit such derived systems. There are examples in section 4 to which previous results on the central limit theorem are not applicable, but our extended central limit theorem does apply.
\end{abstract}

\section{INTRODUCTION}

Let $T$ be a noninvertible nonsingular transformation on a nonatomic probability space $(X, \mathcal{F}, \nu)$. We assume that there exists a countable generating partition $Q=\left\{X_{a}\right\}_{a \in I}$ of $X$ such that for each $X_{a} \in Q,\left.T\right|_{X_{a}}: X_{a} \rightarrow T X_{a}$ is invertible and nonsingular. Nonhyperbolic maps that we shall consider admit indifferent periodic points (see the definition in section 3) and do not necessarily satisfy the Markov property with respect to $Q$ but satisfy the FRS condition (see the definition in section 21) from which we can obtain nice countable state symbolic dynamics which provide natural Markovization of $(T, X, Q)([29])$. The purpose of this paper is to study for such intermittent maps the limiting behaviour of ergodic sums $\sum_{i=0}^{n-1} f T^{i}$ of observables $f$ with respect to $T$-invariant measures $\mu \leq \nu$ and the asymptotic behaviour of Perron-Frobenius operators. Failure of hyperbolicity causes interesting statistical phenomena. In fact, $\mu$ is not necessarily finite and admits an unbounded density $d \mu / d \nu(28,29,33])$. Also we can easily find multi-dimensional intermittent maps $T$ which do not expand distances (i.e., $d(T x, T y) / d(x, y)$ can be strictly smaller than 1 on an open neighbourhood of the indifferent periodic points) even if $Q$ is generating. The failure of the uniformly expanding property causes crucial difficulties in applying previous results on the CLT. In this paper we shall prove an extended central limit theorem in such a nonhyperbolic situation. More specifically, our method is based on the existence of a derived system $\left(T^{*}, Q^{*}, \mu^{*}\right)$ which satisfies Renyi's condition (see section 2) and the Markov property. To establish the central limit theorem for $(T, \mu)$, we clarify the speed of $L^{1}$-convergence of the iterated Perron Frobenius operator for $\left(T^{*}, \mu^{*}\right)$. Then applying [14] and [16] gives

Received by the editors February 20, 1998.

2000 Mathematics Subject Classification. Primary 11K50, 11K55, 28D05, 58F03, 58F11, $58 \mathrm{~F} 15$. 
the CLT for the derived system $\left(T^{*}, \mu^{*}\right)$ which allows us to establish the CLT for $(T, \mu)$ by using the method in [7]. The standard approach for getting exponential convergence and exponential decay of correlations which is based on the IonescuTulcea-Marinescu (ITM) theorem ([15]) appeals to the decay of Lipschitz constant of local inverses. On the other hand, our result on $L^{1}$-convergence does not require this property. This is the point of departure from previous results for piecewise $C^{2}$-expanding maps, and for Gibbs Markov maps (see [3, 5]; cf. [9], 20]). The central limit theorem result of Aaronson, Denker and Urbanksi (ADU) in [7] relied on the high rates of continued fraction mixing for $\left(T^{*}, \mu^{*}\right)$ obtained from the ITM theorem, and so is not applicable to a two-dimensional intermittent map which we shall consider in section 4. In fact, the map admits $\left(T^{*}, \mu^{*}\right)$ for which we have no evidence of decay of Lipschitz constant of local inverses. This is the reason we need the extended CLT, and our results on $L^{1}$-convergence give stretched exponential mixing coefficients for $\left(T^{*}, \mu^{*}\right)$, which is enough for the extended CLT. We should remark that our rates of $L^{1}$-convergence for $\left(T^{*}, \mu^{*}\right)$ are similar to Kuzmin's rates of unifrom convergence for the Gauss transformation.

The paper is organized as follows. In section 2 we collect preliminary results for $T$ satisfying Renyi's condition, and by applying [18] we establish an $L^{1}$-convergence theorem of $\left\{P_{\mu}^{n} f\right\}$ for piecewise Lipschitz continuous functions $f$ with reasonable rates. In section 3 we establish the extended central limit theorem in case $\mu(X)<\infty$. In case $\mu(X)=\infty$ we obtain non-central limit theorems by applying [7] and give a large class of Darling-Kac sets. Proofs are postponed to the Appendix. Furthermore, for $T$ admitting infinite invariant measures we shall give the rates of uniform convergence of the normalized sum of $\left\{P_{\mu}^{n} 1_{A}\right\}$ for Darling-Kac sets $A$ (1]-3]). In section 4, our results in sections 213 apply to three examples admitting indifferent periodic points. The first example is a two-dimensional number theoretical transformation which admits an infinite absolutely continuous invariant measure and is not a Markov map. Our observation allows us to obtain a large class of Darling-Kac sets and non-central limit theorems. The second example is a local skew product map related to the Diophantine approximation problem in the inhomogeneous linear class which admits a finite absolutely continuous invariant measure with unbounded density. The Lipschitz constant of local inverses does not necessarily decay so that the previous results on the CLT are not applicable, i.e., we have a crucial difficulty in establishing high rates of decay of mixing coefficients for $\left(T^{*}, \mu^{*}\right)$, which was assumed in the Aaronson-Denker-Urbanski CLT ([7]). On the other hand, our extended CLT is applicable to this example. The last example is a one-parameter family of maps of an interval with an indifferent fixed point at which the order of tangency determines their statistical properties.

\section{Piecewise invertible systems With FRS SATISFYING RENYI'S CONDITION}

Let $(X, \mathcal{F}, \nu)$ be a nonatomic probability space. Suppose that $T: X \rightarrow X$ is a nonsingular transformation with respect to $\nu$ (i.e., $\nu T^{-1} \sim \nu$ ) and that there exists a countable measurable partition $Q=\left\{X_{a}\right\}_{a \in I}$ of $X$, generating $\mathcal{F}$ and satisfying the local invertibility property, i.e., for every $X_{a} \in Q, \nu\left(X_{a}\right)>0$ and $\left.T\right|_{X_{a}}: X_{a} \rightarrow T X_{a}$ is an invertible map satisfying $\frac{\left.d(\nu T)\right|_{X_{a}}}{d \nu \mid X_{a}}>0$ ( $\nu$-a.e.). Such a system $(T, X, Q)$ is called a piecewise invertible system. Let $Q^{(n)}=\bigvee_{i=0}^{n-1} T^{-i} Q$. We let $X_{a_{1} \ldots a_{n}}$ denote a set $X_{a_{1}} \cap T^{-1} X_{a_{2}} \cap \ldots \cap T^{-(n-1)} X_{a_{n}} \in Q^{(n)}$ of positive 
$\nu$-measure, and call such a set a cylinder of rank $n . \mathcal{L}^{(n)}$ denotes the family of all cylinders $X_{a_{1} \ldots a_{n}}$ and $\mathcal{L}=\bigcup_{n=1}^{\infty} \mathcal{L}^{(n)}$. Let $\mathcal{A}_{n}$ be the set of all admissible sequences of length $n$, i.e., $\mathcal{A}_{n}=\left\{a_{1} \ldots a_{n}: X_{a_{1} \ldots a_{n}} \in \mathcal{L}^{(n)}\right\}$ and $\mathcal{A}=\bigcup_{n=1}^{\infty} \mathcal{A}_{n}$. We assume further:

(Finite Range Structure) $\mathcal{U}=\left\{T^{n} X_{a_{1} \ldots a_{n}}: \forall X_{a_{1} \ldots a_{n}} \in \mathcal{A}_{n}, \forall n>0\right\}$ consists of only finitely many subsets of $X$ with positive $\nu$ measure.

In [7] the piecewise invertible system was called a Markov fibred system if $Q$ is a Markov partition. Let $\mathcal{V}$ be a finite partition generated by $\mathcal{U}$.

Proposition 2.1. Let $(T, X, Q, \mathcal{U})$ be a piecewise invertible system with $F R S$ satisfying the following condition.

(C-1) $\forall U \in \mathcal{U}, \exists X_{a_{1} \ldots a_{s}} \subset U$ such that $T^{s} X_{a_{1} \ldots a_{s}}=X$.

Then $(T, X, Q \vee \mathcal{V}, T(Q \vee \mathcal{V}))$ is an aperiodic Markov fibred system.

Proof. It follows from Theorem 10.1 in [29] that $Q \vee \mathcal{V}$ is a Markov partition for $T$ and $T(Q \vee \mathcal{V}) \subset \sigma(\mathcal{V})$, where $\sigma(\mathcal{V})$ denotes the $\sigma$-algebra generated by $\mathcal{V}$. Aperiodicity of $Q \vee \mathcal{V}$ easily follows from (C-1).

For each $\left(a_{1} \ldots a_{n}\right) \in \mathcal{A}$, we denote the local inverse $\left(\left.T^{n}\right|_{X_{a_{1} \ldots a_{n}}}\right)^{-1}: T^{n} X_{a_{1} \ldots a_{n}}$ $\rightarrow X_{a_{1} \ldots a_{n}}$ by $\psi_{a_{1} \ldots a_{n}}$ and denote $\frac{d\left(\nu \psi_{a_{1} \ldots a_{n}}\right)}{d \nu}(x)\left(=\left(\frac{d\left(\nu T^{n}\right)}{d \nu} \psi_{a_{1} \ldots a_{n}} x\right)^{-1}\right)$ by $\psi_{a_{1} \ldots a_{n}}^{\prime}$. For a constant $C \geq 1$, we shall define

$$
R(C, T)=\left\{X_{a_{1} \ldots a_{n}} \in \mathcal{L}: \sup _{x, y \in T^{n} X_{a_{1} \ldots a_{n}}} \frac{\psi_{a_{1} \ldots a_{n}}^{\prime}(x)}{\psi_{a_{1} \ldots a_{n}}^{\prime}(y)}<C\right\} .
$$

Definition. If there exists a constant $C>1$ such that $R(C, T)=\mathcal{L}$, then we say that $T$ satisfies Renyi's condition.

Definition. The piecewise invertible system $(T, X, Q, \mathcal{U})$ is called continued fraction mixing with respect to the $T$-invariant measure $\mu \sim \nu$, if $\exists 0<C^{\prime}<\infty$ such that

$$
\mu\left(E \cap T^{-n} F\right) \leq C^{\prime} \mu(E) \mu(F)\left(\forall n \in \mathbf{N}, \forall E \in \bigvee_{i=0}^{n-1} T^{-i} Q, \forall F \in \mathcal{F}\right),
$$

and $\exists n_{1} \in \mathbf{N},\left\{\epsilon_{n}\right\}_{n \geq n_{1}}, \epsilon_{n} \rightarrow 0$ as $n \rightarrow \infty$ such that $\forall k \in \mathbf{N}, \forall E^{\prime} \in \bigvee_{i=0}^{k-1} T^{-i} Q$, $\forall F \in \mathcal{F}, \forall n \geq n_{1}$,

$$
\left(1-\epsilon_{n}\right) \mu\left(E^{\prime}\right) \mu(F) \leq \mu\left(E^{\prime} \cap T^{-(k+n)} F\right) \leq\left(1+\epsilon_{n}\right) \mu\left(E^{\prime}\right) \mu(F) .
$$

Theorem 2.1. Let $(T, X, Q, \mathcal{U})$ be a piecewise invertible system with FRS satisfying Renyi's condition and the condition (C-1). Then

1. There exists an ergodic, exact $T$-invariant probability measure $\mu$ equivalent to $\nu$ with $d \mu / d \nu \in\left[G^{-1}, G\right](\exists 0<G<\infty)$.

2. $(T, X, Q \vee \mathcal{V}, T(Q \vee \mathcal{V}))$ is a continued fraction mixing with respect to $\mu$.

Proof. The proof of 1 can be found in [17]. Since (C-1) gives aperiodicity, the Markov condition and Renyi's condition allow us to apply [8] to establish 2 (cf. [7]). 
For $\left(a_{1} \ldots a_{n}\right) \in \mathcal{A}_{n}$ we denote $d\left(\mu \psi_{a_{1} \ldots a_{n}}\right) / d \mu$ by $v_{a_{1} \ldots a_{n}}^{\prime}$. Here $a(n)$ stands for $\left(a_{1} \ldots a_{n}\right) \in \mathcal{A}_{n}$. Let $P_{\nu}: L^{1}(\nu) \rightarrow L^{1}(\nu)$ and $P_{\mu}: L^{1}(\mu) \rightarrow L^{1}(\mu)$ be the Perron-Frobenius operators with respect to $\nu, \mu$ respectively, i.e.,

$$
\begin{aligned}
& P_{\nu} f(x)=\sum_{a \in I} \psi_{a}^{\prime}(x) f\left(\psi_{a}(x)\right) 1_{T X_{a}}(x)\left(f \in L^{1}(\nu)\right), \\
& P_{\mu} f(x)=\sum_{a \in I} v_{a}^{\prime}(x) f\left(\psi_{a}(x)\right) 1_{T X_{a}}(x)\left(f \in L^{1}(\mu)\right) .
\end{aligned}
$$

Theorem 2.2. Let $(T, X, Q, \mathcal{U})$ be a piecewise invertible system with FRS satisfying (C-1). Assume further that

(C-2) $\exists\left\{\delta_{a(n)}\right\}_{a(n) \in \mathcal{A}_{n}}$ with

$$
\sum_{a(n) \in \mathcal{A}_{n}} \delta_{a(n)}<\infty \text { s.t. } \delta_{a(n)}\left(\inf _{x \in T^{n} X_{a(n)}} \psi_{a(n)}^{\prime}(x)\right)^{-1}
$$

is bounded from above uniformly in $a(n) \in \mathcal{A}_{n}, n>0$ and

$$
\left|\psi_{a(n)}^{\prime}(x)-\psi_{a(n)}^{\prime}(y)\right| \leq \delta_{a(n)} d(x, y)(\forall x, y \in V \in \mathcal{V}) .
$$

Then $h \equiv d \mu / d \nu$ is piecewise Lipschitz continuous with respect to $\mathcal{V}$, i.e.,

$$
\exists 0<L_{h}<\infty,|h(x)-h(y)| \leq L_{h} d(x, y)(\forall x, y \in V \in \mathcal{V}) .
$$

Proof. The condition (C-2) allows us to have Renyi's condition and further uniformly boundness and uniformly Lipschitz continuity of $\left\{P_{\nu}^{n} 1\right\}_{n>0}$ with respect to $\mathcal{V}$. The standard argument allows us to establish $(\mathrm{H})$.

Definition. We say that a sequence $\left\{A_{n}\right\}_{n} \geq 1$ decays stretched exponentially if $\exists 0<\eta<1, \exists 0<\delta<1$ such that $A_{n} \leq O\left(\eta^{n^{\delta}}\right)$.

Theorem 2.3 (Rates of $L^{1}$-convergence to the equilibrium state). Let (T, X, Q, $\mathcal{U})$ be a Markov fibred system with FRS satisfying (C-1), (C-2). Assume further that

(C-3) $\sigma(n)=\sup _{X_{a_{1} \ldots a_{n}} \in \mathcal{L}^{(n)}} \operatorname{diam} X_{a_{1} \ldots a_{n}} \rightarrow 0$ as $n \rightarrow \infty$.

Let $f \in L^{1}(\mu)$ with $\int f d \mu=0$ satisfy the following condition.

(C-4) There exist $0<L_{f}<\infty$ and $\theta>0$ such that

$$
\operatorname{var}_{k}(f) \equiv \sup _{X_{b_{1} \ldots b_{k}} \in \mathcal{L}^{(k)}} \sup _{x, y \in X_{b(k)}}|f(x)-f(y)| \leq L_{f} \sigma(k)^{\theta} .
$$

Then there exist $0<\gamma<1$ and $0<n_{0}<\infty$ such that for $n \geq n_{0}$

$$
\left\|P_{\mu}^{n} f\right\|_{1} \leq\|f\|_{1}\left(O\left(\max \left(\sigma\left(n^{\alpha}\right), \sigma\left(n^{\alpha}\right)^{\theta}\right)\right)+(1-\gamma)^{n^{1-\alpha}}\right)(\forall 0<\alpha<1) .
$$

In particular, if $\sigma(n)$ decays exponentially, then $\left\|P_{\mu}^{n} f\right\|_{1}$ decays stretched exponentially. (Cf. [21, [32].)

\section{Corollary 2.1.}

$$
\begin{gathered}
\left\|P_{\nu}^{n} f-h\left(\int_{X} f d \nu\right)\right\|_{L^{1}(\nu)} \leq\|f\|_{L^{1}(\nu)}\left(O\left(\max \left(\sigma\left(n^{\alpha}\right), \sigma\left(n^{\alpha}\right)^{\theta}\right)\right)+(1-\gamma)^{n^{1-\alpha}}\right) \\
(\forall 0<\alpha<1) .
\end{gathered}
$$

Lemma 2.1. There exist $0<\gamma<1$ and $m_{0}>0$ such that for $\forall g \in L_{1}(\mu)$ with $\int g d \mu=0$ and $\forall m \geq m_{0},\left\|P_{\mu}^{m} E\left(g \mid Q^{(m)}\right)\right\|_{1} \leq(1-\gamma)\|g\|_{1}$. (Here $E_{\mu}\left(g \mid Q^{(m)}\right)$ denotes the conditional expectation of $g$ corresponding to $Q^{(m)}$ with respect to $\mu$.) 
Proof. Define

$$
k_{\mu}(x, z)=\sum_{a(m) \in \mathcal{A}_{m}} \frac{1_{X_{a(m)}(z)}}{\mu\left(X_{a(m)}\right)} P_{\mu}^{m} 1_{X_{a(m)}}(x) .
$$

Then it is easy to see that

$$
P_{\mu}^{m} E\left(g \mid Q^{(m)}\right)(x)=\int_{X} g(z) k_{\mu}(x, z) d \mu(z)
$$

and

$$
\begin{gathered}
k_{\mu}(x, z)=h(x)^{-1} \sum_{a(m) \in \mathcal{A}_{m}} \frac{\psi_{a(m)}^{\prime}(x) h\left(\psi_{a(m)} x\right)}{\mu\left(X_{a(m)}\right)} 1_{X_{a(m)}}(z) \\
=h(x)^{-1} \sum_{a(m) \in \mathcal{A}_{m}} \frac{h\left(\psi_{a(m)} x\right) \psi_{a(m)}^{\prime}(x)}{h\left(y_{a(m)}\right) \nu\left(X_{a(m)}\right)} 1_{X_{a(m)}}(z)
\end{gathered}
$$

where $y_{a(m)} \in X_{a(m)}$. Then Renyi's condition allows us to have

$$
\frac{\psi_{a(m)}^{\prime}(x)}{\nu\left(X_{a(m)}\right)} \geq C^{-1}
$$

so that we have $k_{\mu}(x, z) \geq G^{-1} C^{-1}(1-O(\sigma(m)))$. Since $C \geq 1$ and $G^{-1} \leq$ $\int_{X} h(x) d \nu(x)=1$, we have the constant $0<\gamma<1$ and a sufficiently large number $m_{0}$. To get the bound on $\left\|P_{\mu}^{m} E\left(g \mid Q^{(m)}\right)\right\|_{1}$ we follow the argument in [18] (cf. [10], [19]).

$$
\begin{gathered}
\left\|P_{\mu}^{m} E\left(g \mid Q^{(m)}\right)\right\|_{1} \leq 2 \int_{X} g(z) \int_{P_{\mu}^{m} E\left(g \mid Q^{(m)}\right) \geq 0}\left(k_{\mu}(x, z)-\gamma\right) d \mu(x) d \mu(z) \\
\leq 2 \int_{g \geq 0} g(z)(1-\gamma) d \mu(z)=\|g\|_{1}(1-\gamma) . \quad \square
\end{gathered}
$$

Lemma 2.2. For all $l>0,\left\|P_{\mu}^{l} f-E\left(P_{\mu}^{l} f \mid Q^{(m)}\right)\right\|_{1} \leq\|f\|_{1} O(\sigma(m))+L_{f} \sigma(m+l)^{\theta}$.

Proof. First we note that

$$
\begin{gathered}
\left\|P_{\mu}^{l} f-E\left(P_{\mu}^{l} f \mid Q^{(m)}\right)\right\|_{1} \\
\leq \sum_{a(m) \in \mathcal{A}_{m}} \int_{X} \frac{1_{X_{a(m)}}(x)}{\mu\left(X_{a(m)}\right)} \int_{X_{a(m)}}\left|P_{\mu}^{l} f(x)-P_{\mu}^{l} f(y)\right| d \mu(y) d \mu(x) .
\end{gathered}
$$

Next we see that for all $a(n) \in \mathcal{A}_{n}(\forall n>0)$

$$
\begin{array}{r}
\sup _{X_{b(k)} \in \mathcal{L}^{(k)}} \sup _{x, y \in X_{b(k)}}\left|1-\frac{\psi_{a(n)}^{\prime}(x)}{\psi_{a(n)}^{\prime}(y)}\right| \leq O(\sigma(k)), \\
\sup _{X_{b(k)} \in \mathcal{L}^{(k)}} \sup _{x, y \in X_{b(k)}}\left|1-\frac{h(x)}{h(y)}\right| \leq O(\sigma(k)),
\end{array}
$$

and so

$$
\sup _{X_{a(k)} \in \mathcal{L}^{(k)}} \sup _{x, y \in X_{a(k)}}\left|1-\frac{v_{b(l)}^{\prime}(x)}{v_{b(l)}^{\prime}(y)}\right| \leq O(\sigma(k)) .
$$


Combining the above facts gives for $x, y \in X_{a(m)} \subset V \in \mathcal{V}$

$$
\begin{aligned}
\left|P_{\mu}^{l}(x)-P_{\mu}^{l}(y)\right| \leq & \sum_{b(l): T^{l} X_{b(l)} \supset X_{a(m)}}\left|v_{b(l)}^{\prime}(x) f\left(\psi_{b(l)} x\right)-v_{b(l)}^{\prime}(y) f\left(\psi_{b(l)} y\right)\right| \\
\leq & \sum_{b(l): T^{l} X_{b(l)} \supset X_{a(m)}}\left|v_{b(l)}^{\prime}(x)-v_{b(l)}^{\prime}(y)\right|\left|f\left(\psi_{b(l)} x\right)\right| \\
& +\sum_{b(l): T^{l} X_{b(l)} \supset X_{a(m)}} v_{b(l)}^{\prime}(y)\left|f\left(\psi_{b(l)} x\right)-f\left(\psi_{b(l)} y\right)\right| \\
\leq & \sum_{b(l): T^{l} X_{b(l)} \supset X_{a(m)}} v_{b(l)}^{\prime}(y)\left|f\left(\psi_{b(l)} x\right)\right| O(\sigma(m))+L_{f} \sigma(m+l)^{\theta} .
\end{aligned}
$$

These inequalities allow us to have

$$
\begin{gathered}
\left\|P_{\mu}^{l} f-E\left(P_{\mu}^{l} f \mid Q^{(m)}\right)\right\|_{1} \\
\leq O(\sigma(m)) \sum_{a(m) \in \mathcal{A}_{m}} \int_{X} 1_{X_{a(m)}}(x) P_{\mu}^{l}|f|(x) d \mu(x)+L_{f} \sigma(m+l)^{\theta} \\
=\|f\|_{1} O(\sigma(m))+L_{f} \sigma(m+l)^{\theta} .
\end{gathered}
$$

Proof of Theorem 2.3. Since

$$
\begin{gathered}
\left\|P_{\mu}^{m k} f-\left(P_{\mu}^{m} E\left(\cdot \mid Q^{(m)}\right)\right)^{k} f\right\|_{1} \\
\leq \sum_{j=0}^{k-1}\left\|\left(P_{\mu}^{m} E\left(\cdot \mid Q^{(m)}\right)\right)^{j} P_{\mu}^{m}\left(1-E\left(\cdot \mid Q^{(m)}\right)\right) P^{m(k-j-1)} f\right\|_{1} \\
\leq O\left(\max \left(\sigma(m), \sigma(m)^{\theta}\right)\right) \sum_{j=1}^{k-1}(1-\gamma)^{j}\|f\|_{1},
\end{gathered}
$$

we have

$$
\left\|P_{\mu}^{m k} f\right\|_{1} \leq\|f\|_{1}\left(O\left(\max \left(\sigma(m), \sigma(m)^{\theta}\right)\right) \sum_{j=1}^{k-1}(1-\gamma)^{j}+(1-\gamma)^{k}\right) .
$$

Take $m=\left[n^{\alpha}\right]$ and $k=\left[n^{1-\alpha}\right](0<\alpha<1)$, then we have the desired bound.

Proof of Corollary [2.1] We note that $h \geq G^{-1}>0$ (see Theorem [2.1) and that $P_{\nu}(f)=h P_{\mu}(f / h)$. Since $f / h$ satisfies $(\mathrm{C}-4)$, we have

$$
\left\|P_{\nu}^{n} f-\left(\int_{X} f d \nu\right) h\right\|_{L^{1}(\nu)} \leq\left\|P_{\mu}^{n}(f / h)-\int_{X} f d \nu\right\|_{1}
$$

which gives the desired bound.

Theorem 2.4 (Strongly mixing rates). Let $(T, X, Q, \mathcal{U})$ be a Markov fibred system with FRS satisfying (C-1), (C-2), (C-3). Then we have a constant $0<\Gamma<\infty$ such that $\forall E \in \mathcal{F}, \forall k>0, \forall X_{a(m)} \in \mathcal{L}^{(m)}$,

$$
\begin{aligned}
& \left|\mu\left(T^{-(m+k)} E \cap X_{a(m)}\right)-\mu(E) \mu\left(X_{a(m)}\right)\right| \\
\leq & \Gamma\left(O\left(\sigma\left(k^{\alpha}\right)\right)+(1-\gamma)^{k^{1-\alpha}}\right)(\forall 0<\alpha<1) .
\end{aligned}
$$


Proof. For $X_{a(m)} \in \mathcal{L}$ with $T^{m} X_{a(m)}=X$, we define $f(x) \equiv P_{\mu}^{m}\left(1_{X_{a(m)}}-\mu\left(X_{a(m)}\right)\right)$. Then $\int_{X} f d \mu=0$ and $\forall x, y \in X_{b(k)},|f(x)-f(y)|=\left|v_{a(m)}^{\prime}(x)-v_{a(m)}^{\prime}(y)\right| \leq$ $G^{4} C 3 \sigma(k)$ because of (2.2-1)-(2.2-3). Thus $f$ satisfies the condition (C-4) with $\theta=1$. Applying Theorem 2.3 gives the desired bound.

We denote $\operatorname{Var}_{n}(f)=\int_{X}\left(\sum_{i=0}^{n-1}\left(f T^{i}-\int_{X} f d \mu\right)\right)^{2} d \mu$.

Theorem 2.5 (Central Limit Theorem). Suppose that all conditions of Theorem 2.3 are satisfied. Assume that either of the following conditions is satisfied.

(2.5-1) $f \in L^{2+\epsilon}(\mu)(\exists \epsilon>0)$ and $\sum_{k=1}^{\infty}\left(\max \left(\sigma\left(k^{\alpha}\right), \sigma\left(k^{\alpha}\right)^{\theta}\right)\right)^{\frac{\epsilon}{\epsilon+2}}<\infty$ $(\exists 0<\alpha<1)$.

(2.5-2) $f \in L^{\infty}(\mu)$ and $\sum_{k=1}^{\infty}\left(\max \left(\sigma\left(k^{\alpha}\right), \sigma\left(k^{\alpha}\right)^{\theta}\right)\right)<\infty(\exists 0<\alpha<1)$.

Then $\exists \lim _{n \rightarrow \infty} \frac{\operatorname{Var}_{n}(f)}{n} \equiv c_{f}<\infty$ and if $c_{f} \neq 0$, then $f$ satisfies the CLT, i.e., for any $t \in \mathbf{R}$

$$
\lim _{n \rightarrow \infty} \mu\left(\left\{x \in X: \frac{1}{\sqrt{\operatorname{Var}_{n}(f)}} \sum_{i=0}^{n-1} f T^{i}(x) \leq t\right\}\right)=\frac{1}{\sqrt{2 \pi}} \int_{-\infty}^{t} \exp \left(-\left(u^{2} / 2\right)\right) d u .
$$

Corollary 2.2. If $\sigma(n)=O\left(\eta^{n}\right)(0<\eta<1)$, then $f \in L^{2+\epsilon}(\mu)(\exists \epsilon>0)$ satisfies the CLT.

Proof of Theorem 2.5. Suppose that (2.5-1). Then it follows from Theorem 2.4 that $\left\{f T^{n}\right\}_{n>0}$ is a functional of a strongly mixing sequence satisfying all conditions of Theorem 18.6.2 in [16] which are sufficient for CLT.

Suppose that (2.5-2). Then we have

$$
\sum_{n=0}^{\infty}\left\|P_{\mu}^{n} f\right\|_{2} \leq\|f\|_{1} \sum_{n=0}^{\infty}\left\|P_{\mu}^{n} f\right\|_{1} .
$$

Then applying Theorem 2.3 and [14] allows us to establish the CLT.

\section{LIMIT THEOREMS FOR NONHYPERBOLIC PIECEWISE INVERTIBLE SYSTEMS WITH FRS}

In this section, we establish limit theorems for piecewise invertible systems admitting indifferent periodic points. Our method is based on the existence of a derived system (Jump transformation) to which the previous results can apply. Let $(T, X, Q, \mathcal{U})$ be a piecewise invertible system with FRS. We define inductively, for $n \geq 1$,

$$
\begin{gathered}
\mathcal{D}_{n}=\left\{X_{a_{1} \ldots a_{n}} \in \mathcal{L}: X_{a_{1} \ldots a_{i}} \notin R(C, T),(1 \leq i \leq n)\right\}, \\
D_{n}=\bigcup_{X_{a_{1} \ldots a_{n} \in \mathcal{D}_{n}}} X_{a_{1} \ldots a_{n}}, \\
\mathcal{B}_{n}=\left\{X_{a_{1} \ldots a_{n}} \in \mathcal{L}: X_{a_{1} \ldots a_{n-1}} \in \mathcal{D}_{n-1}, X_{a_{1} \ldots a_{n}} \in R(C, T)\right\}, \\
B_{n}=\bigcup_{X_{a_{1} \ldots a_{n}} \in \mathcal{B}_{n}} X_{a_{1} \ldots a_{n}} .
\end{gathered}
$$

In particular we define $D_{0}=X$. Define a map $T^{*}: \bigcup_{i=1}^{\infty} B_{i} \rightarrow X$ by $T^{*} x=T^{i} x$ for $x \in B_{i}$. Put $X^{*}=X \backslash \bigcup_{m=0}^{\infty} T^{*-m}\left(\bigcap_{n \geq 0} D_{n}\right)$. Then $T^{*}$ restricted to $X^{*}$ gives a transformation of $X^{*}$. Define $I^{*}=\bigcup_{n=1}^{\infty}\left\{\left(a_{1} \ldots a_{n}\right) \in I^{n}: X_{a_{1} \ldots a_{n}} \in \mathcal{B}_{n}\right\}$ and $Q^{*}=\left\{X_{\alpha}\right\}_{\alpha \in I^{*}} \cdot \mathcal{L}^{*(n)}$ and $\mathcal{L}^{*}$ are defined as well as $\mathcal{L}^{(n)}$ and $\mathcal{L}$, with respect to $T^{*}$. 
Put $\mathcal{U}^{*}=\left\{T^{* n} X_{\alpha_{1} \ldots \alpha_{n}}: n>0, X_{\alpha_{1} \ldots \alpha_{n}} \in \mathcal{L}^{*(n)}\right\}$ and let $\mathcal{V}^{*}$ be a finite partition generated by $\mathcal{U}^{*}$. Suppose that the following conditions are satisfied.

(1) (The local Renyi condition).

$$
X_{a(n) b(l)} \in \mathcal{L}, X_{b(l)} \in R(C, T) \text { implies } X_{a(n) b(l)} \in R(C, T) .
$$

(2) (Transitivity) $\forall U \in \mathcal{U}, \exists X_{a_{1} \ldots a_{s}} \subset U$ such that $T^{s} X_{a_{1} \ldots a_{s}}=X$ and $X_{a_{s}} \in$ $R(C, T)$.

Then we can easily verify that $\left(T^{*}, X^{*}, Q^{*}, \mathcal{U}^{*}\right)$ is a piecewise invertible system with FRS satisfying Renyi's condition and $\forall U \in \mathcal{U}^{*}, \exists X_{\alpha_{1} \ldots \alpha_{s}} \in \mathcal{L}^{*}$ such that $X_{\alpha_{1} \ldots \alpha_{s}} \subset U$ and $T^{* s} X_{\alpha_{1} \ldots \alpha_{s}}=X^{*}$. It follows from Theorem 2.1 that the jump system $\left(T^{*}, X^{*}, Q^{*}, \mathcal{U}^{*}\right)$ admits an ergodic exact $T^{*}$-invariant measure $\mu^{*} \sim \nu$ with $d \mu^{*} / d \nu$ bounded away from zero and infinity ([17], [22], [27]). Furthermore the Markovization $\left(T^{*}, X^{*}, Q^{*} \vee \mathcal{V}^{*}, T^{*}\left(Q^{*} \vee \mathcal{V}^{*}\right)\right)$ is a continued fraction mixing with respect to $\mu^{*}([8$, [7] $)$. To obtain a $T$ - invariant measure $\mu$ equivalent to $\nu$, we need to assume

(3) $\lim _{n \rightarrow \infty} \nu\left(D_{n}\right)=0$.

Then the following formula gives an ergodic $\sigma$-finite $T$-invariant measure $\mu$ equivalent to $\nu$.

(I-1) $\mu(A)=\sum_{n=0}^{\infty} \mu^{*}\left(T^{-n} A \cap D_{n}\right) \quad(\forall A \in \mathcal{F}) .($ Cf. [22]-[25], 27].)

Furthermore, we have exactness of $\mu([27$, [29]). The next condition gives a criterion of finiteness of $\mu$.

(4) $\sum_{n=0}^{\infty} \nu\left(D_{n}\right)<\infty$.

In fact, it follows from (I-1) that (4) is equivalent to $\mu(X)<\infty$.

Remark (A). Denote the stopping time with respect to $R(C, T)$ by $\phi(x)=\inf \{n \in$ $\left.\mathbf{N}: X_{a_{1} \ldots a_{n}}(x) \in R(C, T)\right\}$ where $X_{a_{1} \ldots a_{n}}(x)$ denotes the unique cylinder of rank $n$ containing $x$. Then (4) implies finiteness of the first moment of $\phi: E_{\nu}(\phi)=$ $\int_{X} \phi(x) d \nu(x)<\infty$. We will see that finiteness of the second moment of $\phi$ is important for getting a large class of functions in which the CLT holds.

Remark (B). The invariant density $d \mu / d \nu$ is unbounded at periodic points $x_{0}$ (with period $q$ ) satisfying $\frac{d\left(\nu T^{q}\right)}{d \nu}\left(x_{0}\right)=1\left([28-[29])\right.$. We call such a periodic point $x_{0}$ indifferent with respect to $\nu$. In particular, for piecewise $C^{1}$ maps $T$ and for $\nu$ the Lebesgue measure, the definition coincides with the usual one (i.e., $\left|\operatorname{det} D T^{q}\left(x_{0}\right)\right|=$ $1)$.

Remark $(\mathrm{C})$. Let $\mathcal{U}^{*}=\left\{U_{0}, U_{1}, \ldots, U_{N}\right\}$. Even if we weaken the transitivity condition in (2) to

$$
\begin{aligned}
X^{*}= & \bigcup_{i=0}^{N} U_{i} \text { and for each } U_{i}, \exists X_{\alpha}^{(i, j)}\left(s_{j}\right) \subseteq U_{i} \text { s.t. } \\
& T^{* s_{i}} X_{\alpha^{(i, j)}}\left(s_{i}\right)=U_{j}(\text { for } j=0,1, \ldots, N),
\end{aligned}
$$

we still have a $\sigma$-finite ergodic $T^{*}$-invariant measure $\mu^{*}$ equivalent to $\nu$ (see Theorem $1^{*}$ in [17]) and $\left(T^{*}, \mu^{*}\right)$ is exact (see [27]).

For each $\alpha \in I^{*}$ and $\alpha_{1} \ldots \alpha_{n} \in \mathcal{L}^{*(n)}$, we denote $\psi_{\alpha}^{*}=\left(\left.T^{*}\right|_{X_{\alpha}}\right)^{-1}$ and $\psi_{\alpha_{1} \ldots \alpha_{n}}^{*}=$ $\left(\left.T^{* n}\right|_{X_{\alpha_{1} \ldots \alpha_{n}}}\right)^{-1}$ respectively. Define $f^{*}(x) \equiv \sum_{i=0}^{\phi(x)-1} f T^{i}(x)-\phi(x) \int_{X} f d \mu$ and $S_{n} f^{*} \equiv \sum_{i=0}^{n-1} f^{*} T^{* i}$. We denote the variance of $S_{n} f^{*}, \operatorname{Var}\left(S_{n} f^{*}\right)$ by $\sigma_{n}^{2}$. Let $Q^{*(n)}=\bigvee_{i=0}^{n-1} T^{*-i} Q^{*}$ and $\sigma^{*}(n)=\sup _{\mathcal{L}^{*(n)}} \operatorname{diam} X_{\alpha_{1} \ldots \alpha_{n}}$. First we establish the 
central limit theorem in the case $\mu(X)<\infty$. We put $\mu_{0}=\mu / \mu(X)$ and write an integral $\int_{X} g d \mu$ as $E_{\mu}(g)$.

Theorem 3.1 (The central limit theorem). Let $(T, X, Q, \mathcal{U})$ be a Markov fibred system with FRS satisfying (1),(2),(4) and either of the next two conditions.

(3-1-a) $\phi T(x)=\phi(x)-1$ if $\phi(x)>1$.

(3-1-b) $\exists n_{0}>0$ s.t. $B_{n+n_{0}} \subseteq D_{n} \cap T^{-n} B_{n_{0}}(\forall n>0)$.

Suppose that $T^{*}$ satisfies $(\mathrm{C}-2)$,

(3-2) $\sigma^{*}(n)=O\left(\eta^{n}\right)(\exists 0<\eta<1)$,

and $f \in L^{\infty}(\mu)$ satisfies

(3-3) $\exists \theta>0$ and for each $X_{a(n)} \in \mathcal{B}_{n}, \exists 0<L_{f}(a(n))<\infty$ with $L_{f}(\infty) \equiv$ $\sup _{n} \sup _{\mathcal{B}_{n}} \sum_{i=1}^{n} L_{f}\left(a_{i} \ldots a_{n}\right)<\infty$ such that for $x, y \in X_{b(k)} \cap T^{n} X_{a(n)}$

$$
\left|f\left(\psi_{a(n)} x\right)-f\left(\psi_{a(n)} y\right)\right| \leq L_{f}(a(n))\left(\operatorname{diam} X_{b(k)}\right)^{\theta} .
$$

Assume further either of the next two conditions.

(3-4-a) $\int_{X} f d \mu=0$, and $(\operatorname{supp} f) \cap\left(\bigcap_{n \geq 0} D_{n}\right)=\emptyset$.

(3-4-b) $\exists \epsilon>0$ with $E_{\nu}\left(\phi^{2+\epsilon}\right)<\infty$.

Then $\exists \lim _{n \rightarrow \infty} \sigma_{n}^{2} / n \equiv c_{f}<\infty$ and if $c_{f} \neq 0$, then $f$ satisfies the central limit theorem, i.e., for any $t \in \mathbf{R}$

$$
\lim _{n \rightarrow \infty} \mu_{0}\left(\left\{x: \sqrt{\frac{\mu(X)}{c_{f} n}} \sum_{i=0}^{n-1}\left(f T^{i}(x)-\int_{X} f d \mu_{0}\right) \leq t\right\}\right)=\frac{1}{\sqrt{2 \pi}} \int_{-\infty}^{t} \exp \left(-u^{2} / 2\right) d u .
$$

We use the following sequence of lemmas.

Lemma 3.1. (3-3) implies that

$$
\left\|f^{*}-E_{\mu^{*}}\left(f^{*} \mid Q^{*(n)}\right)\right\|_{\infty} \leq L_{f^{*}} \sigma^{*}(n-1)\left(\exists 0<L_{f^{*}}<\infty\right) .
$$

Lemma 3.2. If $f \in L^{\infty}(\mu)$ satisfies (3-4-a), then $f^{*} \in L^{\infty}\left(\mu^{*}\right)$.

Lemma 3.3. If $f \in L^{\infty}(\mu)$ satisfies (3-4-b), then $f^{*} \in L^{2+\epsilon}\left(\mu^{*}\right)$.

Applying Theorem 2.5 allows us to have the following two results.

Lemma 3.4. Suppose that (3-3) is satisfied and $T^{*}$ satisfies (C-2) and

$$
\sum_{k=1}^{\infty} O\left(\max \left(\sigma^{*}\left(k^{\alpha}\right), \sigma^{*}\left(k^{\alpha}\right)^{\theta}\right)\right)<\infty(\exists 0<\alpha<1) .
$$

Then $f^{*} \in L^{\infty}\left(\mu^{*}\right)$ satisfies the $C L T$.

Lemma 3.5. Suppose that (3-3) is satisfied and $T^{*}$ satisfies (C-2) and

$$
\sum_{k=1}^{\infty} O\left(\max \left(\sigma^{*}\left(k^{\alpha}\right), \sigma^{*}\left(k^{\alpha}\right)^{\theta}\right)^{\frac{\epsilon}{\epsilon+2}}\right)<\infty(\exists 0<\alpha<1) .
$$

Then $f^{*} \in L^{2+\epsilon}\left(\mu^{*}\right)$ satisfies the CLT.

Let $\sigma\left(\mathcal{F}_{0}^{m}\right)(\exists m>0)$ denote the $\sigma$-algebra generated by cylinders of rank $m$. The next theorem is obtained by applying Corollary 7.3 in [7] immediately.

Theorem 3.2. Let $(T, X, Q, \mathcal{U})$ be a Markov fibred system with FRS satisfying (1),(2),(4) and either (3-1-a) or (3-1-b). If $E_{\nu}\left(\phi^{2}\right)<\infty$ and $f \in L^{\infty}(\mu)$ is measurable with respect to $\sigma\left(\mathcal{F}_{0}^{m}\right)(\exists m>0)$, then $\lim _{n \rightarrow \infty} \operatorname{Var}_{n}\left(f^{*}\right)=\infty$ implies 
$\operatorname{Var}_{n}\left(f^{*}\right)=n \rho(n)$, where $\rho(n)$ is a slowly varying function, and we have for any $t \in \mathbf{R}$

$\lim _{n \rightarrow \infty} \mu_{0}\left(\left\{x: \sqrt{\mu(X) / \sigma_{n}^{2}} \sum_{i=0}^{n-1}\left(f T^{i}(x)-\int_{X} f d \mu_{0}\right) \leq t\right\}\right)=\frac{1}{\sqrt{2 \pi}} \int_{-\infty}^{t} \exp \left(-u^{2} / 2\right) d u$.

Remark (D). Under the condition (3-1-a), $E_{\nu}\left(\phi^{2}\right)<\infty$ implies finiteness of the second moment of the return time function to $B_{1}$ defined by $\phi_{B_{1}}(x)=\inf \{n \geq 1$ : $\left.T^{n} x \in B_{1}\right\}$.

Proposition 3.1 (Failure of the square integrability). Let $E_{\nu}\left(\phi^{2}\right)=\infty$. Then for $f \in L^{\infty}(\mu)$ with $\mu(f) \neq 0$, we have $f^{*} \notin L^{2}\left(\mu^{*}\right)$. If $f \in L^{\infty}(\mu)$ satisfies $\mu(f)=0$, and $\exists g \in L^{\infty}(\mu)$ with $\int g d \mu \neq 0$ satisfying $f=g-\int_{X} g d \mu$, then $f^{*} \notin L^{2}\left(\mu^{*}\right)$.

Corollary 3.1. For $f \in L^{\infty}(\mu)$ with $\mu(f) \neq 0, E_{\nu}\left(\phi^{2}\right)=\infty$ if and only if $f^{*} \notin$ $L^{2}\left(\mu^{*}\right)$.

In most cases, multi-dimensional number theoretical transformations admitting indifferent periodic points satisfy $E_{\nu}\left(\phi^{2}\right)=\infty$ even if $E_{\nu}(\phi)<\infty$. Without finiteness of the second moment of $\phi$, we may have a crucial difficulty in establishing the CLT for $f$ because of non-hyperbolicity. Now we ask:

Question (1) Suppose that $E_{\nu}\left(\phi^{2}\right)=\infty$. Let $f \in L^{\infty}(\mu)$ be a piecewise Hölder continuous function satisfying either $\mu(f) \neq 0$ or $($ supp $f) \cap \bigcap_{n \geq 0} D_{n}$ $\neq \emptyset$. Then do we still have the central limit theorem?

In [5], the domain of attraction of stable laws had been obtained for Gibbs Markov maps. Under our weaker assumptions, nothing is known.

Question (2) Suppose that $E_{\nu}\left(\phi^{2}\right)=\infty$. Can we determine the class of functions in the domain of attraction of a stable law?

We recall that the following characterization of the stable law: if $\left\{X_{i}\right\}$ i.i.d. random variables obey a stable law $\mathcal{G}_{\alpha}$ with index $\alpha \in(0,2)$, then the normalizing sequence should be $a_{n}=n^{1 / \alpha} \rho(n)$, where $\rho$ is a slowly varying function. We will see some relation between the index $\alpha$ of the normalizing sequence $a_{n}$ and the degree of nonhyperbolicity in section 4 .

In the rest of this section, we discuss statistical properties in the case $\mu(X)=\infty$. For $A \in \mathcal{F}$ with $0<\mu(A)<\infty, \phi_{A}$ denotes the return time function of $T$ on $A$. It follows from the conservativity and ergodicity (see 29]) that we can define the induced transformation $T_{A}$ on $A$ by $T_{A} x=T^{\phi_{A}(x)} x$. We denote the Markov partition $Q \vee \mathcal{V}$ by $\mathcal{M}$.

Definition. A set $A \in \mathcal{F}$ with $0<\mu(A)<\infty$ is called a Darling-Kac set for $T$ if there exists $\left\{a_{n}(T)\right\}_{n \geq 1}$ such that $\lim _{n \rightarrow \infty} \frac{1}{a_{n}(T)} \sum_{k=1}^{n-1} P_{\mu}^{k} 1_{A}=\mu(A)$ uniformly on A.

First we state limit theorems:

Theorem 3.3. Let $(T, X, Q, \mathcal{U})$ be a piecewise invertible system with FRS satisfying the conditions (1)-(3). Suppose that $\int \phi(x) d \nu(x)\left(=\sum_{n=0}^{\infty} \nu\left(D_{n}\right)\right)=\infty$ which implies $\mu(X)=\infty$. Then $A \in R(C, T) \cap \mathcal{M}$ is a Darling-Kac set and if the return sequence $a_{n}(T)$ is regulary varying with index $\alpha \in[0,1]$, then we have the following.

(3.3-1) For all $f \in L^{1}(\mu), \mu(f) \neq 0, \forall P \leq \mu$, the distribution of $\frac{\sum_{i=0}^{n-1} f T^{i}}{\Gamma(1+\alpha) a_{n}(T)}$ is weakly convergent to the dual to the one-sided stable law $\mathcal{G}_{\alpha}$ with index $\alpha \in[0,1]$ which is called the Mittag-Leffler distribution $\mathcal{M L}_{\alpha}$ of exponent $\alpha$, 
(3.3-2) Assume that $\alpha>0$. For $B \in \mathcal{F}$ with $0<\mu(B)<\infty, \frac{\sum_{i=0}^{n-1} \phi_{B}\left(T_{B}^{k}\right)}{b_{n}}$ is weakly convergent to the one-sided stable law $\mathcal{G}_{\alpha}$ with index $\alpha \in(0,1]$, where $b_{n}$ is the asymptotic inverse of $a_{n} \Gamma(1+\alpha)$.

(3.3-3) When $\alpha=1$ and $a_{n}(T) \sim n \rho(n)$,

$$
\exists K_{T} \in(0,1] \text { s.t. } \varlimsup_{n \rightarrow \infty} \frac{\sum_{i=0}^{n-1} f T^{i}}{n \rho(n / \log \log n)}=K_{T} \int f d \mu
$$

a.e. for $f \in L_{+}^{1}(\mu)$. Moreover, if $\rho(n / \log \log n) \sim \rho(n)$ (as $\left.n \rightarrow \infty\right)$, then

$$
\varlimsup_{n \rightarrow \infty} \frac{\sum_{i=0}^{n-1} f T^{i}}{n \rho(n)}=\int f d \mu
$$

a.e. for $f \in L_{+}^{1}(\mu)$.

Remark (E). Since $\mathcal{M} \mathcal{L}_{1}$ is concentrated in 1, (3.3-1) for the case $\alpha=1$ implies that the system is homogeneous in the sense of Aaronson ([1]).

As we have already shown in section 2, the Markovization of $(T, X, Q, \mathcal{U})$ satisfying (C-1) is an aperiodic Markov fibred system. Furthermore, we can easily verify the following.

Lemma 3.6. Let $(T, X, Q, \mathcal{U})$ be a piecewise invertible systems satisfying (1)-(3). Then $(T, X, \mathcal{M}, T \mathcal{M})$ is an aperiodic Markov fibred system with FRS satisfying the local Renyi condition.

Proof of Theorem [3.3. Using Lemma [3.6, we can apply Theorem 3.1 in [7] to $(T, X, \mathcal{M}, T \mathcal{M})$ so that any $A \in R(C, T) \cap \mathcal{M}$ is a Darling-Kac set for $T$ whose return time process is continued fraction mixing and $T$ is pointwise dual ergodic, i.e., for any $f \in L^{1}(\mu) \lim _{n \rightarrow \infty} \frac{1}{a_{n}(T)} \sum_{k=0}^{n-1} P_{\mu}^{k} f=\int_{X} f d \mu$ a.e. (cf. 2]). Then (3.3-1)-(3.3-2) follow from the results which were established in 11, 4] and [34] (cf. [7]).

The main contribution to infinite invariant measure case is the following result which gives a large class of Darling-Kac sets.

Proposition 3.2. Suppose that $A$ is a union of finitely many cylinders belonging to $R(C, T) \cap \mathcal{M}$ over which the induced system satisfies (2)*. Then $A$ is a Darling-Kac set.

Proof of Proposition 3.2. Define a countable partition $Q_{A}$ of $A$ by

$$
Q_{A}=\left\{X_{a^{0}\left(n_{0}\right) b(l) a^{1}\left(n_{1}\right)} \in \mathcal{L}: X_{a^{i}\left(n_{i}\right)} \subset A(i=1,0), X_{b(l)} \in \mathcal{L}^{(l)}, l \geq 0\right\} .
$$

Then $T_{A}\left(Q_{A}\right)=: \mathcal{U}_{A}=\left\{X_{a(n)} \subseteq A\right\}$. We can easily see that $\left\{T_{A}, A, Q_{A}, \mathcal{U}_{A}\right\}$ is a piecewise invertible system with FRS satisfying Renyi's condition, the Markov condition and further aperiodicity. Then the bounded metric distortion (Lemma 4.1 in [29]) allows us to apply [8] which gives continued fraction mixing property for $T_{A}$. The desired results follows from [2] immediately.

The most difficult point in applying the limit theorems (3.3-1)-(3.3-3) to practical examples is to show that $a_{n}(T)$ is regularly varying. It can be done by computing the asymptotic rate explicitly. It was shown in [2] that $a_{n}(T)$ is given by 
$a_{n}(A) / \mu(A)^{2}$ with a Darling-Kac set $A$ and Theorem 6.1 in 29 insists that there is an increasing sequence $W(n)$ such that

$$
\mu\left(\bigcup_{l=0}^{n} T^{-l} B\right)=: W_{B}(n) \sim W(n) \text { as } n \rightarrow \infty
$$

for all $A^{\prime} \in \mathcal{F}_{0}, B \in A^{\prime} \cap \mathcal{F}, \nu(B)>0$, where $\mathcal{F}_{0}$ denotes the ring generated by $R(C, T)$. Combining the asymptotic renewal equations (see [2]) and the Karamata Tauberian Theorem gives the next relation between $a_{n}(T)$ and $W(n)$ in the case when $W(n)$ is regularly varying with index $\alpha \in[0,1]$,

$$
\frac{a_{n}(T) W(n)}{n} \sim \frac{1}{\Gamma(1+\alpha) \Gamma(2-\alpha)} \text { as } n \rightarrow \infty \text {. }
$$

The next result was proved in 7] (Lemma 5.2), which will be used in section 4.

Proposition 3.3. Under (3-1-a) we have

$$
W_{B_{l}}(n)=\mu\left(\bigcup_{i=1}^{n} B_{i}\right)+O(1) \quad(\forall l>0)
$$

and

$$
\mu\left(\bigcup_{i=1}^{n} B_{i}\right)=\int_{X} \min \{\phi, n\} d \nu .
$$

Remark (F). Examples (1) and (3) in section 4 satisfy (3-1-a).

For Darling-Kac sets $A$, we have a variety of bounds on the rate of uniform convergence of $\frac{1}{a_{n}(T)} \sum_{k=1}^{n} \mathcal{P}_{\mu}^{k} 1_{A}$ to $\mu(A)$ with respect to $\|\cdot\|_{L^{\infty}(A)}$ which allows us to establish a log-averaged ergodic theorem. Let $\mu_{A}, \nu_{A}$ denote the conditional measures associated to $A$, respectively.

Theorem 3.4. Let $(T, X, Q, \mathcal{U})$ be a piecewise invertible system with FRS satisfying the conditions (1)-(3). Suppose that $A=\bigcup_{i=1}^{r} A_{i}, A_{i} \in \mathcal{M} \cap R(C, T)$, $A \subset \bigcup_{i=1}^{m} B_{i}(\exists m>0)$ and the induced system over $A$ satisfies $(2)^{*}$. Then for every $\delta_{1}, \delta_{2}>0$ with $0<\delta_{1}, \delta_{2}<1, \exists 0<F_{1}, F_{2}<\infty$ s.t.

$$
c_{n}(A) \equiv\left\|\frac{\sum_{k=1}^{n} \mathcal{P}_{\mu}^{k} 1_{A}(x)}{a_{n}(T)}-\mu(A)\right\|_{L^{\infty}(A)}
$$

$$
\leq F_{1}\left(F_{2} n^{1-\delta_{1}} / a_{n}(T)+\epsilon_{\left[n^{\left(1-\delta_{1}\right)\left(1-\delta_{2}\right)}\right]+1}+W_{m}\left(1+\epsilon_{1}^{2}\right)\left[n^{\left(1-\delta_{1}\right)\left(1-\delta_{2}\right)}\right] e_{n}\left(A, \delta_{1}, \delta_{2}\right)\right)
$$

where

$$
\begin{gathered}
W_{m}=\sup _{\bigcup_{i=1}^{m} B_{i}} d \mu / d \nu \\
e_{n}\left(A, \delta_{1}, \delta_{2}\right) \equiv \nu_{A}\left(A^{c} \cap T^{-1} A^{c} \cap \ldots \cap T^{-\left[n^{\delta_{2}\left(1-\delta_{1}\right)}\right]+1} A^{c}\right)
\end{gathered}
$$

and $\epsilon_{i}$ is the continued fraction mixing coefficient for $\left(T_{A}, \mu_{A}\right)$. (Here $[y]$ denotes the integer part of $y$.) In particular, we have the following:

(3-4-2) Denote the maximum of ranks of the cylinders $A_{i}(1 \leq i \leq r)$ by $\mathrm{J}$. If $\nu(A)>1-L / C_{J}$, where $L=\min _{U \in \mathcal{U}} \nu(U)$ and

$$
C_{J}=\sup _{X_{a(J)} \in \mathcal{L}^{(J)}} \frac{\sup _{x \in T^{J} X_{a(J)}} \psi_{a(J)}^{\prime}(x)}{\inf _{x \in T^{J} X_{a(J)}} \psi_{a(J)}^{\prime}(x)},
$$

then $e_{n}\left(A, \delta_{1}, \delta_{2}\right)$ is bounded by a sequence $\left(\frac{C_{J}(1-\nu(A))}{L}\right)^{\left[n^{\left.\left(1-\delta_{1}\right) \delta_{2} / J\right]}\right.}$ which decays stretched exponentially. 
(3-4-3) If $A=\bigcup_{i=1}^{m} B_{i}$, then $e_{n}\left(A, \delta_{1}, \delta_{2}\right) \leq \nu_{A}\left(D_{\left[n^{\left.\left(1-\delta_{1}\right) \delta_{2}\right]}\right.}\right)$.

Corollary 3.2. In the case of (3-4-2), the last term of RHS of (3-4-1) decays faster than any polynomial decay. If $\epsilon_{n}$ decays stretched exponentially, then we have $c_{n}(A) \leq a_{n}(T)^{-1}$. In particular, when (4) is valid, $c_{n}(A) \leq n^{-1}$.

Remark (G). As we will see later in section 4 , in most cases, $\epsilon_{n}$ decays at least stretched exponentially. For a Bernoulli $(L=1)$ linear $\left(C_{J}=1\right)$ map, $(3-4-2)$ is valid for all $A$ with $0<\nu(A)$ which is a finite union of cylinders belonging to $\mathcal{M} \cap R(C, T)$.

Remark (H). Theorem 3.4 can be used to obtain a log-averaged ergodic theorem, i.e., almost sure convergence of $\frac{1}{\log N} \sum_{n=1}^{N} \frac{1}{n a_{n}(T)} \sum_{k=1}^{n} f T^{k}\left(f \in L^{1}(\mu)\right)$. Following [6], if $a_{n}(T)$ is regularly varying with positive index, one of the sufficient conditions for establishing the theorem can be described as $c_{n}(A) \leq O\left(\frac{1}{\log N^{\gamma}}\right)$, for some $\gamma>0$.

Proof of Theorem 3.4. The inequalities (1-2) in the proof of Theorem 2.1 in [2] allow us to have for $1 \leq p \leq q \leq n-1$

$$
\begin{gathered}
\left\|\frac{\sum_{k=1}^{n} \mathcal{P}_{\mu}^{k} 1_{A}}{\sum_{k=1}^{n} \mu_{A}\left(T^{-k}(A)\right)}-1\right\|_{L^{\infty}(A)} \leq \epsilon_{p+1}+\left(1+\epsilon_{1}\right)^{2} \mu_{A}\left(\left\{\sum_{k=0}^{p-1} \phi_{A} T_{A}^{k} \geq q\right\}\right) \\
+\frac{\left(1+\epsilon_{1}^{2}\right) q+p}{\sum_{k=1}^{n} \mu_{A}\left(T^{-k} A\right)}
\end{gathered}
$$

and so we obtain a bound on $\left\|\frac{\sum_{k=1}^{n} \mathcal{P}_{\mu}^{k} 1_{A}}{a_{n}(T)}-\mu(A)\right\|_{L^{\infty}(A)}$. Next we observe that

$$
\left\{x \in A: \sum_{k=0}^{p-1} \phi_{A} T_{A}^{k}(x) \geq q\right\} \subset \bigcup_{k=1}^{p}\left\{x \in A: \phi_{A}\left(T^{k} x\right) \geq q / p\right\} .
$$

In fact, if not i.e., $\forall k, \phi_{A}\left(T^{k} x\right)<q / p$ and $\sum_{k=0}^{p-1} \phi_{A} T_{A}^{k}(x) \geq q$, we have a contradiction immediately. Furthermore, it is easy to see that

$$
\mu_{A}\left(\bigcup_{i=1}^{p-1}\left\{x \in A: \phi_{A}\left(T^{i} x\right) \geq[q / p]+1\right\}\right) \leq p \mu_{A}\left(A^{c} \cap T^{-1} A^{c} \cap \ldots \cap T^{-\left(\left[\frac{q}{p}\right]\right)+1} A^{c}\right) .
$$

Put

Taking

$$
d_{n}(A)=\frac{\left(1+\epsilon_{1}^{2}\right) q+p}{\sum_{k=0}^{n-1} \mu_{A}\left(T^{-k} A\right)}
$$

we have

$$
q=n^{1-\delta_{1}} \quad\left(0<\delta_{1}<1\right), \quad p=q^{1-\delta_{2}} \quad\left(0<\delta_{2}<1\right)
$$

$$
d_{n}(A) \sim \frac{q}{a_{n}(T)}=n^{1-\delta_{1}} / a_{n}(T), \text { as } n \rightarrow \infty
$$

because of the ergodicity. Combining these observations we come to the desired inequality. To see (3-4-2), first we shall show for the case when $A=X_{a}$. Then noting

$$
\nu_{A}\left(A^{c} \cap T^{-1} A^{c} \cap \ldots \cap T^{-\left[\frac{q}{p}\right]+1} A^{c}\right)=\sum_{b_{1} \neq a} \sum_{b_{2} \neq a} \ldots \sum_{b_{\left[\frac{q}{p}\right]} \neq a} \nu_{A}\left(X_{b_{1} \ldots b_{\left[\frac{q}{p}\right]}}\right)
$$

gives (3-4-2). Even if $A$ is not a single cylinder, the similar argument allows us to have the desired result. 


\section{EXAMPLES AND APPLiCATIONS}

We apply our results in previous sections to piecewise $C^{1}$ intermittent maps for which reference measures $\nu$ are the normalized Lebesgue measures.

Example (1). (A two dimensional map related to uniform distribution of a cylinder flow) Let $X=\{(x, y): 0<x \leq 1,0 \leq y \leq 1\}$ and $T$ is defined by

$$
T(x, y)=\left(-\frac{1}{x}-\left[-\frac{1}{x}\right], \frac{y}{x}-\left[\frac{y}{x}\right]\right) .
$$

The first component of $T$ is known as a map related to negative continued fraction which admits a $\sigma$-finite infinite absolutely continuous invariant measure with respect to the Lebesgue measure $([23])$. Let $a(x)=-[-1 / x]$, and $b(x, y)=[y / x]$. The index set $I$ is given by

$$
I=\{(a, b) \in \mathbf{N} \times(\mathbf{N} \cup\{0\}): a \geq 2, a>b\} .
$$

The partition $Q=\left\{X_{(a, b)}\right\}_{(a, b) \in I}$ is defined as follows:

$$
(x, y) \in X_{(a, b)} \text { iff } a(x)=a \text { and } b(x, y)=b .
$$

It is easy to see that $\mathcal{U}$ consists of $U_{0}=X$ and $U_{1}=\{(x, y) \in X: x+y \leq 1\}$ and that $Q$ is not Markov but satisfies the FRS condition. In [17, (1)-(3) were verified so that $T$ admits a $\sigma$-finite infinite ergodic invariant measure equivalent to $\nu$ the Lebesgue measure and it was shown that $\mathcal{D}_{n}$ is the set of cylinders touching $\{1\} \times[0,1]$ which consists of indifferent fixed points $(|\operatorname{det} D T(x, y)|=1)$, i.e.,

$$
\begin{gathered}
\mathcal{D}_{n}=\left\{X_{\left(\left(2, b_{1}\right),\left(2, b_{2}\right), \ldots\left(2, b_{n}\right)\right)}: b_{1}=b_{2}=\ldots=b_{n}=0\right. \\
\text { or } \left.b_{i}=1(\exists i) \text { and } b_{j}=0(\forall j \neq i)\right\} .
\end{gathered}
$$

The validity of (3-1-a) is verified immediately. By Theorem 9.1 in 29] these indifferent fixed points are s-singular points of $\frac{d \mu}{d \nu}$ so that $\mu(X)=\infty$. In fact the invariant density was given in [17]:

$$
\frac{d \mu}{d \nu}(x, y)= \begin{cases}\frac{2-x}{2(1-x)^{2}} & \text { if } x+y<1 \\ \frac{1}{2(1-x)} & \text { if } x+y>1 .\end{cases}
$$

By Propositions $3.2[3.3$ we have the wandering rate (cf. [29]) $W(n)=\log (n+1)$ which is slowly varying and $a_{n}(T) \sim \frac{n}{\log (n+1)}$ as $n \rightarrow \infty$. Hence (3.3-1)-(3.3-3) in Theorem 3.3 can be applied to this example.

Example (2). (A local skew product two-dimensional map which is related to Diophantine approximation in inhomogeneous linear class) It is well-known that Gauss transformation induces a best approximation to an irrational number $x \in$ $(0,1)$, i.e., for any $N>0, \min _{0 \leq q \leq N}|q x-p|=\left|q_{n} x-p_{n}\right|$ where $n$ is chosen as $q_{n} \leq N \leq q_{n+1}$ and $\left(q_{n}, p_{n}\right)$ are the principal convergents of $x$. Let $X=\{(x, y) \in$ $\left.\mathbf{R}^{2}: 0 \leq y \leq 1,-y \leq x \leq-y+1\right\}$. Define $T$ on $X$ by

$$
T(x, y)=(1 / x-[(1-y) / x]+[-(y / x)],-[-(y / x)]-(y / x)) .
$$

The local skew product map $T$ induces a best approximation to irrational numbers $(x, y) \in X$ for the inhomogeneous linear form, $\min _{(u, v), 1<|u|<N}|u||u x+y-v|$ (see [23]). We denote

$$
a(x, y)=[(1-y) / x]-[-(y / x)], b(x, y)=-[-(y / x)]
$$

$a_{n}(x, y)=a\left(T^{n-1}(x, y)\right), b_{n}(x, y)=b\left(T^{n-1}(x, y)\right)$, and define $I=\left\{(a, b) \in \mathbf{Z}^{2}:\right.$ $a>b>0$, or $a<b<0\}$. Let $X_{(a, b)}=\{(x, y) \in X \mid a(x, y)=a, b(x, y)=b\}$. 
Then $Q=\left\{X_{(a, b) \in I}\right\}$ is a countable partition of $X$ and on each cylinder $X_{(a, b)} T$ is given by $T(x, y)=((1 / x)-a,-(y / x)+b)$. Since $T^{n} X_{\left(a_{1}, b_{1}\right), \ldots\left(a_{n}, b_{n}\right)}=X$ for all $X_{\left(a_{1}, b_{1}\right) \ldots\left(a_{n}, b_{n}\right)} \in \mathcal{L}, T$ is a Bernoulli map. Let us define inductively $T^{n}(x, y)=$ $\left(X_{n}, Y_{n}\right)$ and $q_{n}=a_{n} q_{n-1}+q_{n-2}\left(q_{0}=1, q_{-1}=0\right), p_{n}=a_{n} p_{n-1}+p_{n-2}\left(p_{0}=\right.$ $\left.0, p_{-1}=1\right)$. Then we obtain the following formulas:

$$
\begin{aligned}
& \psi_{\left(a_{1}, b_{1}\right), \ldots\left(a_{n}, b_{n}\right)}(x, y)=\left(\frac{p_{n}+p_{n-1} x}{q_{n}+q_{n-1} x}, \sum_{k=1}^{n}(-1)^{k-1} x_{0}(x) x_{1}(x) \ldots x_{k-1}(x) b_{k}\right. \\
& \left.+(-1)^{n} x_{0}(x) x_{1}(x) \ldots x_{n-1}(x) y\right) \\
& x=\frac{p_{n}+p_{n-1} X_{n}}{q_{n}+q_{n-1} X_{n}} \\
& y=\sum_{k=1}^{n}\left(q_{k-1} x-p_{k-1}\right) b_{k}+\frac{Y_{n}}{q_{n}+q_{n-1} X_{n}} .
\end{aligned}
$$

The above formulas give the following inequalities which will be used later:

$$
\begin{aligned}
\left|x-\frac{p_{n}}{q_{n}}\right| & \leq \frac{1}{\left|q_{n}\right|} \frac{1}{\left|q_{n}\right|-\left|q_{n-1}\right|} \\
\left|y-\sum_{k=1}^{n}\left(q_{k-1} x-p_{k-1}\right) b_{k}\right| & \leq \frac{1}{\left|q_{n}\right|-\left|q_{n-1}\right|}=\frac{1}{\left|q_{n}\right|\left(1-\left|q_{n-1}\right| /\left|q_{n}\right|\right)} .
\end{aligned}
$$

Observe that

$$
\left|\operatorname{det} D \psi_{\left(a_{1}, b_{1}\right) \ldots\left(a_{n}, b_{n}\right)}(x, y)\right|=\frac{1}{\left|q_{n}\right|^{3}\left|1+\left(q_{n-1} / q_{n}\right) x\right|^{3}},
$$

and that

$$
q_{n-1} / q_{n}=\frac{1}{a_{n}+\frac{1}{a_{n-1}+\ldots \frac{1}{a_{1}}}} \in[-1,1] .
$$

Then we can choose $3^{3}$ as the local Renyi constant, and $\mathcal{D}_{k}$ and $\mathcal{B}_{k}$ as follows (cf. [28]):

$$
\begin{gathered}
\mathcal{D}_{k}=\{\underbrace{X_{(2,1)(-2,-1)(2,1)(-2,-1) \ldots}}_{k}, X_{(-2,-1)(2,1)(-2,-1)(2,1) \ldots}^{(-2, \ldots}, \\
\mathcal{B}_{k}=\left\{X_{\left(a_{1}, b_{1}\right) \ldots\left(a_{k}, b_{k}\right)}: X_{\left(a_{1}, b_{1}\right) \ldots\left(a_{k-1}, b_{k-1}\right)} \in \mathcal{D}_{k-1}, a_{k} \neq \pm 2\right. \\
\text { or } \left.a_{k-1} a_{k}>0 \text { if }\left|a_{k}\right|=2\right\} .
\end{gathered}
$$

In [27]-[28], (1),(2),(4) were verified and the density of the finite $T$-invariant measure with respect to the Lebesgue measure $\nu$ was given by:

$$
h(x, y)=\frac{1}{(2 \log 2)\left(1-x^{2}\right)} .
$$

$h$ admits singular points $(1,0)$ and $(-1,1)$ which are indifferent periodic points with period 2 (i.e., $\left|\operatorname{det} D T^{2}(1,0)\right|=\left|\operatorname{det} D T^{2}(-1,1)\right|=1$ ). It is easy to see that $T$ does not expand distances on neighbourhoods of the indifferent periodic points, e.g., let $(x, y)=(1-\epsilon, 0)$ and $\left(x^{\prime}, y^{\prime}\right)=(1-\epsilon / 2, \epsilon / 2)$. Then

$$
d\left(\psi_{(-2,-1)}(x, y), \psi_{(-2,-1)}\left(x^{\prime}, y^{\prime}\right)\right) \geq \frac{\sqrt{2}}{1+\epsilon} d\left((x, y),\left(x^{\prime}, y^{\prime}\right)\right) .
$$


It follows from this inequality that the map $T$ is not uniformly expanding and we have no evidence of decay of Lipschitz constant of local inverses $\left\{\psi_{\alpha_{1} \ldots \alpha_{n}}^{*}\right.$ : $\left.\left(\alpha_{1} \ldots \alpha_{n}\right) \in I^{* n}, n \geq 1\right\}$ for $T^{*}$. For this reason we cannot apply the CLT for Gibbs Markov maps to $\left(T^{*}, \mu^{*}\right)$. $(3-1-b)$ is verified immediately. We note that $X_{\left(a_{1}, b_{1}\right), \ldots\left(a_{n}, b_{n}\right)} \in R(C, T)$ implies $\left|a_{n}\right| \geq 3$ or $a_{n-1} a_{n}>0$ with $\left|a_{n}\right|=2$ and so $\left|q_{n}\right| /\left|q_{n-1}\right| \geq 2$. Then (5) and the following observations allow us to have the fact: (6) $\operatorname{diam} X_{\alpha_{1} \ldots \alpha_{k}} \leq$ constant $\times 2^{-(2 k-1)}$ for any cylinder $X_{\alpha_{1} \ldots \alpha_{k}}$ with respect to $T^{*}$. Let

$$
\begin{gathered}
\alpha_{1}=\left(\left(a_{1}, b_{1}\right), \ldots,\left(a_{n_{1}}, b_{n_{1}}\right)\right), \alpha_{2}=\left(\left(a_{n_{1}+1}, b_{n_{1}+1}\right), \ldots\left(a_{n_{1}+n_{2}}, b_{n_{1}+n_{2}}\right)\right) \\
\ldots, \alpha_{k}=\left(\left(a_{n_{1}+\ldots+n_{k-1}+1}, b_{n_{1}+\ldots+n_{k-1}+1}\right), \ldots,\left(a_{n}, b_{n}\right)\right)
\end{gathered}
$$

where $n=n_{1}+\ldots+n_{k}$. Then

$$
\frac{q_{n-1}}{q_{n}}=\frac{1}{a_{n}+\frac{1}{a_{n-1}+\frac{1}{\ldots a_{n-n_{k}+1}+\frac{q_{n-n_{k}-1}}{q_{n-n_{k}}}}}}
$$

and

$$
\left|\frac{q_{n-n_{k}}}{q_{n}}\right|=\left|\frac{q_{n-1}}{q_{n}}\right|\left|\frac{q_{n-2}}{q_{n-1}}\right| \ldots\left|\frac{q_{n-n_{k}}}{q_{n-n_{k}+1}}\right| \leq\left(\frac{1}{2}\right)^{2} .
$$

Inductively we have

$$
\left|\frac{1}{q_{n}}\right| \leq\left(\frac{1}{2}\right)^{2}\left|\frac{1}{q_{n-n_{k}}}\right| \leq \ldots \leq\left(\frac{1}{2}\right)^{2 k} .
$$

The fact (6) together Theorem 2.4 allows us to establish the CLT for $f$ satisfying (3-3),(3-4-a) and strongly mixing coefficients for $T^{*}$ which decays stretched exponentially. As we have mentioned in section 1 applying Corollary 2.1 with $\alpha=1 / 2$ gives the similar rates to Kuzmin's rates of uniform convergence for Gauss trtansformation. The condition (3-4-b) fails because of $\nu\left(D_{n}\right)=O\left(n^{-2}\right)$. We let $L_{\left(a_{1}, b_{1}\right), \ldots,\left(a_{n}, b_{n}\right)}$ denote the Lipschitz constant of $\psi_{\left(a_{1}, b_{1}\right), \ldots,\left(a_{n}, b_{n}\right)}$. Then we can observe that for each $X_{\left(a_{1}, b_{1}\right), \ldots,\left(a_{n}, b_{n}\right)} \in \mathcal{B}_{n}, L_{\left(a_{1}, b_{1}\right), \ldots,\left(a_{n}, b_{n}\right)} \leq O\left(n^{-1}\right)$. It follows from this fact that if $f$ is a piecewise Hölder continuous function with exponent $\theta$, then (3-3) is valid when $\theta>1$. Next we suppose that $f$ is not Hölder continuous but satisfies the following conditions: $\exists 0<\theta<\infty$ such that for each $X_{\left(a_{1}, b_{1}\right), \ldots,\left(a_{n}, b_{n}\right)} \in \mathcal{B}_{n}, \exists 0<\Gamma_{f}\left(\left(a_{1}, b_{1}\right), \ldots,\left(a_{n}, b_{n}\right)\right)<\infty$ satisfying

$$
\begin{gathered}
\left|f((x, y))-f\left(\left(x^{\prime}, y^{\prime}\right)\right)\right| \\
\leq \Gamma_{f}\left(\left(a_{1}, b_{1}\right), \ldots,\left(a_{n}, b_{n}\right)\right)\left(\operatorname{diam} X_{\left(a_{1}, b_{1}\right), \ldots,\left(a_{n}, b_{n}\right),\left(c_{1}, d_{1}\right), \ldots\left(c_{k}, d_{k}\right)}\right)^{\theta} \\
\left(\forall(x, y),\left(x^{\prime}, y^{\prime}\right) \in X_{\left.\left(a_{1}, b_{1}\right), \ldots,\left(a_{n}, b_{n}\right)\left(c_{1}, d_{1}\right), \ldots\left(c_{k}, d_{k}\right)\right), \text { and }}\right. \\
\sup _{X_{\left(a_{1}, b_{1}\right), \ldots,\left(a_{n}, b_{n}\right)} \in \mathcal{B}_{n}} \Gamma_{f}\left(\left(a_{1}, b_{1}\right), \ldots,\left(a_{n}, b_{n}\right)\right) \leq O\left(n^{\theta-(1+\alpha)}\right)(\exists \alpha>0) .
\end{gathered}
$$

Then taking $L_{f}\left(\left(a_{1}, b_{1}\right), \ldots,\left(a_{n}, b_{n}\right)\right)=\Gamma_{f}\left(\left(a_{1}, b_{1}\right), \ldots,\left(a_{n}, b_{n}\right)\right)\left(L_{\left(a_{1}, b_{1}\right), \ldots,\left(a_{n}, b_{n}\right)}\right)^{\theta}$ allows us to have (3-3). Let $p_{1}$ be the natural projection: $(x, y) \rightarrow x$. If $F$ : $p_{1}(X) \rightarrow \mathbf{R}$ is a piecewise Hölder continuous function with respect to $p_{1} Q$ with exponent $\theta>1 / 2$, then $f \equiv F p_{1}$ satisfies (3-3). In fact, we can easily see that

$$
d\left(p_{1} \psi_{\left(a_{1}, b_{1}\right), \ldots\left(a_{n}, b_{n}\right)}(x, y), p_{1} \psi_{\left(a_{1}, b_{1}\right), \ldots\left(a_{n}, b_{n}\right)}\left(x^{\prime}, y^{\prime}\right)\right) \leq \frac{\left|x-x^{\prime}\right|}{\left|q_{n}\right|^{2}\left(1-\left|q_{n-1}\right| /\left|q_{n}\right|\right)^{2}},
$$


and that $\left|q_{n}\right| \geq O(n)$ and $\left|q_{n-1}\right| /\left|q_{n}\right| \leq 1 / 2$ for $X_{a_{1} \ldots a_{n}} \in \mathcal{B}_{n}$. From these observations we have

$$
\left|f\left(\psi_{\left(a_{1}, b_{1}\right), \ldots\left(a_{n}, b_{n}\right)}(x, y)\right)-f\left(\psi_{\left(a_{1}, b_{1}\right), \ldots\left(a_{n}, b_{n}\right)}\left(x^{\prime}, y^{\prime}\right)\right)\right| \leq O\left(n^{-2 \theta}\right)\left|x-x^{\prime}\right|^{\theta}
$$

and $L_{f}(\infty)=\sum_{i=1}^{\infty} O\left(i^{-2 \theta}\right)<\infty$ for $\theta>1 / 2$.

Example (3) (A one-parameter family of maps on the interval $[0,1]$ ). Let $X=$ $[0,1]$ and for $\beta$ with $0<\beta$ define

$$
T_{\beta}(x)=\left\{\begin{array}{cl}
\frac{x}{\left(1-x^{\beta}\right)^{1 / \beta}} & \text { on } X_{0}=\left[0,(1 / 2)^{1 / \beta}\right), \\
\frac{x}{1-(1 / 2)^{1 / \beta}} & \text { on } X_{1}=\left[(1 / 2)^{1 / \beta}, 1\right] .
\end{array}\right.
$$

The map $T_{\beta}$ has an indifferent fixed point 0 . Let

$$
\mathcal{D}_{n}=\left\{X_{n}^{0 \ldots 0}\right\} \quad \text { and } \quad \mathcal{B}_{n}=\left\{X_{n}^{0 \ldots 0}\right\} .
$$

Then $2^{1+1 / \beta}$ can be taken as the local Renyi constant and $T$ admits a $\sigma$-finite conservative exact invariant measure $\mu$ equivalent to the Lebesgue measure $\nu$ (27][29]). In case $0<\beta<1, \sum_{n=0}^{\infty} \nu\left(D_{n}\right)=\sum_{n=0}^{\infty} 1 /(n+1)^{1 / \beta}<\infty$ which implies that $\mu$ is finite. Several statistical properties were established in 27 -30. For $\beta \geq 1$, $\sum_{n=0}^{\infty} \nu\left(D_{n}\right)=\infty$, which implies $\mu(X)=\infty$. In this case, $T_{\beta}^{\prime \prime}(0)$ is finite and so $T_{\beta}$ belongs to the class of one-dimensional maps with infinite invariant measures which Thaler studied in [24]-[25]. Since $\frac{d \mu}{d \nu}(x) \sim$ constant $\times x^{-\beta}$ near 0 (cf. [22]), Proposition 3.3 allows us to estimate the wandering rates $W(n)$ and the return time sequence $a_{n}(T)$ (up to constants) as follows:

$$
\begin{aligned}
& (\beta=1) W(n) \sim \log n, \quad a_{n}(T) \sim n / \log n . \\
& (\beta>1) W(n) \sim n^{1-1 / \beta}, \quad a_{n}(T) \sim n^{1 / \beta} .
\end{aligned}
$$

Thus for $\beta=1, a_{n}(T)$ is regularly varying with index 1 , and for $\beta>1, a_{n}(T)$ is regularly varying with index $1 / \beta(<1)$. Hence we can apply (3.3-1)-(3.3-3) in Theorem 3.3 to this example.

Next we study the central limit theorem for $0<\beta<1$. Since $\left|\psi_{\alpha_{1} \ldots \alpha_{k}}^{*}{ }^{\prime}(x)\right| \leq$ $\left|\psi_{1}\right|^{k}=\left(1-(1 / 2)^{1 / \beta}\right)^{k}(\forall x \in[0,1]), \sup _{X_{\alpha_{1} \ldots \alpha_{k}} \in \mathcal{L}^{*(k)}} \operatorname{diam} X_{\alpha_{1} \ldots \alpha_{k}}$ decays exponentially as $k \rightarrow \infty$. Hence Theorem 3.1 can be applied to $T_{\beta}$ with $\beta>0$. Observe that $E_{\nu}\left(\phi^{2+\epsilon}\right)=\sum_{n>0} n^{2+\epsilon} \nu\left(B_{n}\right)$ and $\nu\left(B_{n}\right) \sim O\left(n^{-(1+1 / \beta)}\right)$. These observations allow us to have the following:

$(0<\beta<1 / 2) E_{\nu}\left(\phi^{2+\epsilon}\right)<\infty(\exists \epsilon>0)$ and so we can apply Theorem 3.1 . $(1 / 2 \leq \beta<1) E_{\nu}\left(\phi^{2}\right)=\infty$ and so we can establish the CLT for $f$ satisfying (3-3) and (3-4-a).

\section{Appendix. Proofs of the Results in section 3}

Proof of Lemma 3.1. First we note that for $x, y \in X_{\alpha_{1} \ldots \alpha_{n}} \in \mathcal{L}^{*(n)}$

$$
\begin{aligned}
\mid f^{*}(x) & -f^{*}(y)\left|\leq \sum_{i=0}^{\left|\alpha_{1}\right|-1}\right| f\left(\psi_{\alpha_{1}^{i+1} \ldots \alpha_{1}^{\left|\alpha_{1}\right|}} \psi_{\alpha_{2} \ldots \alpha_{n}}^{*} x^{\prime}\right)-f\left(\psi_{\alpha_{1}^{i+1} \ldots \alpha_{1}^{\left|\alpha_{1}\right|}} \psi_{\alpha_{2} \ldots \alpha_{n}}^{*} y^{\prime}\right) \mid \\
& \leq \sum_{i=0}^{\left|\alpha_{1}\right|-1} L_{f}\left(\alpha_{1}^{i+1} \ldots \alpha_{1}^{\left|\alpha_{1}\right|}\right)\left(\operatorname{diam} X_{\alpha_{2} \ldots \alpha_{n}}\right)^{\theta} \leq L_{f}(\infty)\left(\sigma^{*}(n-1)\right)^{\theta}
\end{aligned}
$$


where $x^{\prime}=T^{* n} x$ and $y^{\prime}=T^{* n} y, \alpha_{1}=\alpha_{1}^{1} \ldots \alpha_{1}^{\left|\alpha_{1}\right|} \in I^{*}$ and $\left|\alpha_{1}\right|$ denotes the length of $\alpha_{1}$. Then by the mean value theorem we have that

$$
\begin{gathered}
\left|f^{*}(x)-\sum_{Y \in \mathcal{Q}^{*(n)}} \frac{\int_{Y} f^{*}(z) d \mu^{*}(z)}{\mu^{*}(Y)} 1_{Y}(x)\right| \\
\leq \sup _{Y \in \mathcal{Q}^{*(n)}} \sup _{x^{\prime}, y^{\prime} \in Y}\left|f^{*}\left(x^{\prime}\right)-f^{*}\left(y^{\prime}\right)\right| \leq L_{f}(\infty)\left(\sigma^{*}(n-1)\right)^{\theta} .
\end{gathered}
$$

Proof of Lemma 3.2. It is obtained immediately from the definition of $f^{*}$.

Proof of Lemma 3.3. Since $\int \phi^{2+\epsilon} d \nu=\sum_{l=1}^{\infty} l^{2+\epsilon} \nu\left(B_{l}\right)$, the following inequlity allows us to have the desired result.

$$
\int f^{* 2+\epsilon} d \nu \leq \sum_{l=1}^{\infty} \int_{B_{l}} l^{2+\epsilon}\left(\sup _{x \in D_{l}^{c}}|f(x)|+\int f d \mu\right)^{2+\epsilon} d \nu .
$$

Proof of Proposition 3.1 . The first assertion is obtained from the following inequalities.

$$
\begin{gathered}
\int f^{* 2} d \nu \leq \sum_{l=1}^{\infty} l^{2} \nu\left(B_{l}\right)\left(\sup _{\bigcup_{i=1}^{l} B_{i}}|f|\right)^{2}+2 \mu(f) \sum_{l=1}^{\infty} l^{2} \nu\left(B_{l}\right) \sup _{\bigcup_{i=1}^{l} B_{i}}|f|+\mu(f)^{2} E_{\nu}\left(\phi^{2}\right), \\
\int f^{* 2} d \nu \geq \mu(f)^{2} E_{\nu}\left(\phi^{2}\right)-2 \mu(f) \sum_{l=1}^{\infty} l \nu\left(B_{l}\right) \sup _{\bigcup_{i=1}^{l} B_{i}}|f| .
\end{gathered}
$$

The second assertion is obvious from the fact that $f^{*}(x)=g^{*}(x)$.

Proof of Theorem 3.1. It follows from (3-2) and Lemma 3.1 that

$$
\left(\left\|f^{*}-E_{\mu^{*}}\left(f^{*} \mid \mathcal{Q}^{*(n)}\right)\right\|_{\infty}\right)^{2+\epsilon} \times n^{2+\epsilon}
$$

is uniformly bounded from above. Now we can apply Lemma 7.4 in [7] directly. Suppose either (3-4-a) or (3-4-b). Then it follows from Lemmas 3.4 and 3.5 that $f^{*}$ satisfies the CLT with respect to $\mu^{*}$. Since the rest of the proof is based on the proof of Theorem 7.2 in [7], we just give a sketch of the proof. Let $0<\delta<\psi_{1}^{*}$, where $\left\{\psi_{n}^{*}\right\}_{n \geq 0}$ are the $\psi$-mixing coefficients for $\left(T^{*}, \mu^{*}\right)$ and $\eta>0$ be choosen as $1 / \sqrt{2 \pi} \int_{t}^{t+4 \eta} \exp \left(-u^{2} / 2\right) d u<\delta$. Put $g \equiv f-\mu_{0}(f), \sigma_{n}=\sqrt{\sigma_{n}^{2}}$ and note that $\mu\left(\left\{\sqrt{\mu(X)} / \sigma_{n} \sum_{l=0}^{n} g T^{l} \leq t\right\}\right) \leq(\mathrm{A})+(\mathrm{B})+(\mathrm{C})$, where

$$
\begin{gathered}
(\mathrm{A}):=\mu\left(\left\{\sqrt{\mu(X)} / \sigma_{n} \sum_{l=N_{k_{1}+1}}^{N_{n}} f^{*} T^{* l} \leq t+3 \eta\right\}\right), \\
(\mathrm{B}):=\mu\left(\left\{\sqrt{\mu(X)} / \sigma_{n} \sum_{l=0}^{k_{1}}\left|g T^{l}\right| \geq \eta\right\}\right), \\
(\mathrm{C}):=\mu\left(\left\{\sqrt{\mu(X)} / \sigma_{n}\left|\sum_{l=k_{1}+1}^{n} g T^{l}-\sum_{l=M_{k_{1}}+1}^{M_{n}} g T^{l}\right| \geq 2 \eta\right\}\right)
\end{gathered}
$$

for some $k_{1}>0$, where

$$
N_{n}(x)=\min \left\{l: \sum_{i=0}^{l} \phi\left(T^{* i} x\right) \geq n\right\}, \quad M_{n}(x)=\sum_{i=0}^{N_{n}(x)} \phi\left(T^{* i} x\right) .
$$


Then for an appropriate large number $k_{1}>0$, we can bound (A), (B) and (C) by $\delta+1 / \sqrt{2 \pi} \int_{-\infty}^{u} \exp \left(-v^{2} / 2\right) d v, \delta$ and $2 \delta$ respectively. Hence we have

$$
\mu\left(\left\{\sqrt{\mu(X)} / \sigma_{n} \sum_{l=0}^{n} g T^{l} \leq t\right\}\right) \leq 4 \delta+1 / \sqrt{2 \pi} \int_{-\infty}^{u} \exp \left(-v^{2} / 2\right) d v
$$

for sufficiently large $n>0$. In fact, if $\sigma_{n} \rightarrow \infty$, we can obtain $(B) \leq \delta$ and the fact $\mu(X)=\sum_{n=1}^{\infty} \mu\left(B_{n}\right)<\infty$ and (3-1-a) gives

(D)

$$
\begin{gathered}
\mu\left(\left\{\sqrt{\mu(X)} / \sigma_{n} \sum_{n+1}^{M_{n}(x)} g T^{l}(x) \geq \eta\right\}\right) \\
\leq \mu\left(\left\{M_{n}(x)-n \geq \frac{\sigma_{n} n}{\sqrt{\mu(X)}\|g\|_{\infty}}, T^{n} x \in B_{M_{n}(x)-n}\right\}\right)=\sum_{p \geq p_{0}} \mu\left(B_{p}\right)<\delta
\end{gathered}
$$

where $p_{0}=\left[\frac{\sigma_{n} n}{\sqrt{\mu(X)}\|g\|_{\infty}}\right]$. Even if we replace (3-1-a) by (3-1-b), as far as $p_{0}>n_{0}$ we still have the same upper bound as one of (D). For bounding (A), we use ergodicity so that $\frac{N_{n}(x)}{n} \rightarrow \frac{1}{\mu(X)}(n \rightarrow \infty)$, the formula (I-1) of $\mu$ and the central limit theorem for $\left(T^{*}, \mu^{*}\right)$.

Proof of Theorem 3.2. Since $\left(T^{*}, Q^{*}\right)$ is aperiodic and $f^{*} \in L^{2}\left(\mu^{*}\right)$, we can apply Corollary 7.3 in [7].

\section{ACKNOWLedgement}

The author would like to thank M. Denker for valuable advice, and J. Aaronson for helpful discussions.

\section{REFERENCES}

[1] J. Aaronson. The asymptotic distribution behavior of transformations preserving infinite measures. J. Analyse Math. 39 (1981), 203-234. MR 82m:28030

[2] J. Aaronson. Random f-expansions. The Annals of Probability 14 (1986), 1037-1057. MR 87k:60057

[3] J. Aaronson. An Introduction to Infinite Ergodic Theory (1997), Amer. Math. Soc. MR 99k:28025

[4] J. Aaronson and M. Denker. Lower bounds for partial sums of certain positive stationary process, almost everywhere convergence, Proceedings (G.A. Edgar and L. Sucheston, eds.), Academic Press, (1989) 1-9. MR 91e:28012

[5] J. Aaronson and M. Denker. Local limit theorems for Gibbs Markov maps. Preprint.

[6] J. Aaronson, M. Denker and A. Fisher. Second order ergodic theorems for ergodic transformations of infinite measure spaces, Proc. Amer. Math. Soc. 114 (1992), 115-127. MR 92e:28007

[7] J. Aaronson, M. Denker and M. Urbanski. Ergodic Theory for Markov fibred systems and parabolic rational maps. Trans. Amer. Math. Soc. 337 (1993), 495-548. MR 94g:58116

[8] R. Bradley. On the $\psi$-mixing condition for stationary random sequences. Trans. Amer. Math. Soc. 276 (1983), 55-66. MR 84h:60071

[9] A. Broise. Fractions continues multidimensionelles et lois stables. Bull. Soc. Math. France 124 (1996), 97-139 MR 97h:11081

[10] N. I. Chernov. Limit Theorems and Markov approximations for certain chaotic dynamical systems. Prob. Theor. Relat. Fields. 101 (1995), 321-362. MR 96m:28016

[11] D. A. Darling and M. Kac. On occupation times for Markov processes. Trans. Amer. Math. Soc. 84 (1957), 444-458. MR 18:832a

[12] R. A. Davis. Stable limits for partial sums of dependent random variables. Ann. Probab. 11 (1983), 262-269. MR 84e:60028 
[13] P. Gaspard and X. J. Wang. Sporadicity: between periodic and chaotic dynamical behaviours. Proc. Natl. Acad. Sci. USA 85 (1988) 4591-4595. MR 90f:58115

[14] M. Gordin. Exponentially fast mixing. Dokl. Akad. Nauk SSSR 96 (1971), 1255-1258. MR 43:2752

[15] Ionescu-Tulcea and G. Marinescu. Theorie ergodique pour des classes d'operations non completement continuous. Ann. Math. 47 (1946), 140-147. MR 12:226

[16] I. A. Ibragimov and Y. V. Linnik. Independent and stationary sequences of random variables. Wolters-Noordhoff, Groningen, Netherlands. (1971). MR 48:1287

[17] Sh. Ito and M. Yuri. Number theoretical transformations and their ergodic properties. Tokyo J. Math. 10 (1987) 1-32. MR 88i:28031

[18] C. Liverani. Flows, random perturbations and rate of mixing. Ergodic Theory and Dynamical Systems 18 (1998), 1421-1446. CMP 99:05

[19] C. Liverani, B. Saussall, and S. Vaianti. A probabilistic approach to Intermittency. Preprint.

[20] D. Mayer. Approach to equilibrium for locally expanding maps in $\mathbf{R}^{k}$. Commun. Math. Phys. 95 (1984), 1-15. MR 86d:58069

[21] M. Pollicott. Rates of mixing for potentials of summable variation. Trans. Amer. Math. Soc., to appear. CMP 98:12

[22] F. Schweiger. Some remarks on ergodicity and invariant measures. Michigan Math. J. 22 (1975), 123-131. MR 47:8446

[23] F. Schweiger. Ergodic Theory of Fibred Systems and Metric Number Theory. Oxford University Press. MR 97h:11083

[24] M. Thaler. Estimates of the invariant densities of endomorphisms with indifferent fixed points. Israel J. Math. 37 (1980), 303-314. MR 82f:28021

[25] M. Thaler. Transformations on $[0,1]$ with infinite invariant measures. Israel J. Math. 46 (1983) 67-96. MR 85g:28020

[26] M. S. Waterman, Some ergodic properties of multi-dimensional f-expansions. Z. Wahrsch. Verw. Gebiete 16 (1971), 77-103. MR 44:173

[27] M. Yuri. On a Bernoulli property for multi-dimensional mappings with finite range structure. Tokyo J. Math. 9 (1986) 457-485. MR 88d:28023

[28] M. Yuri. Invariant measures for certain multi-dimensional maps. Nonlinearity 7 (3) (1994), 1093-1124. MR 95c:11101

[29] M. Yuri. Multi-dimensional maps with infinite invariant measures and countable state sofic shifts. Indagationes Mathematicae 6 (1995), 355-383. MR 96j:58059

[30] M. Yuri. On the convergence to equilibrium states for certain nonhyperbolic systems. Ergodic Theory and Dynamical Systems 17, (1997) 977-1000. MR 98f:58155

[31] M. Yuri. Zeta functions for certain nonhyperbolic systems and topological Markov approximations. Ergodic Theory and Dynamical Systems 18 (1998), 1589-1612. CMP 99:05

[32] M. Yuri. Decay of correlations for certain multi-dimensional maps. Nonlinearity 9 (1996), 1439-1461. MR 97i:58100

[33] M. Yuri. Thermodynamic Formalism for certain nonhyperbolic maps. To appear in Ergodic Theory and Dynamical Systems.

[34] R. Zweimuller. Probabilistic properties of dynamical systems with infinite invariant measures, Univ. Salzburg M.Sc. thesis (1995).

Department of Business Administration, Sapporo University, Nishioka, Toyohira-ku, SAPPORO 062, JAPAN

E-mail address: yuri@math.sci.hokudai.ac.jp

E-mail address: yuri@math-ext.sapporo-u.ac.jp 